This is a postprint version of the following published document:

Sánchez-Camacho, A., Pozuelo, J., Mendicuti, F. \& Mattice, W. L. (1997): Intramolecular energy transfer in naphthalene-containing polyesters: Experiment and simulation for model compounds derived from five aliphatic dicarboxylic acids and 2-hydroxynaphthalene. Journal of Fluorescence, 7 (2), pp.: 113-120.

DOI: $10.1007 / \mathrm{BF} 02760502$

(C) Plenum Publishing Corporation, 1997 


\title{
Intramolecular Energy Transfer in Naphthalene-Containing Polyesters: Experiment and Simulation for Model Compounds Derived from Five Aliphatic Dicarboxylic Acids and 2-Hydroxynaphthalene
}

\author{
Ana Sánchez-Camacho, ${ }^{1}$ Javier Pozuelo, ${ }^{1}$ Francisco Mendicuti, ${ }^{1}$ and Wayne L. Mattice ${ }^{2}$
}

\begin{abstract}
Steady-state fluorescence depolarization measurements and molecular dynamics simulations have been used to study the efficiency of nonradiative intramolecular singlet energy transfer between 2 naphthoxy groups, denoted $\mathrm{N}$, in model compounds for polyesters derived from 2,6-dihydroxynaphthalene and aliphatic dicarboxylic acids. The five bichromophoric compounds studied are the diesters abbreviated as N-OOC- $\left(\mathrm{CH}_{2}\right)_{n}-\mathrm{COO}-\mathrm{N} ; n=2-6$, which are condensation products obtained from 2-naphthol and aliphatic dicarboxylic acids. The anisotropy of the fluorescence of these compounds dispersed in a solid matrix of glassy poly(methyl methacrylate) indicates that there is nonradiative singlet energy transfer between naphthoxy groups. The efficiency of this transfer depends on $n$. A theoretical treatment using molecular dynamics simulations for the conformations of the five model compounds has been performed in order to evaluate the parameters related to the efficiency of the transfer. The experimental and theoretical variation of such parameters with $n$ is consistent with the estimated Förster radius of 9-10 $\AA$ for this system.
\end{abstract}

KEY WORDS: Fluorescence anisotropy; intramolecular energy transfer; methylene spacers; naphthalene; polyesters.

\section{INTRODUCTION}

Singlet-singlet nonradiative intramolecular energy transfer of the excited-state energy from a donor (D) to an acceptor (A) and intramolecular excimer formation have been widely used in the characterization of macromolecules containing chromophores. Both ways of deactivating the singlet electronic excited state are strongly dependent on the conformation and the dynamics of the chain, because of the sensitivity to the separation and orientation of the chromophores. ${ }^{(1-4)}$ A conformation

' Departamento de Química Fisica, Universidad de Alcalá, Alcalá de Henares, 28871 Madrid, Spain.

2 Institute of Polymer Science, University of Akron, Akron, Ohio 44325-3909. where two chromophore rings are coplanar and separated by a short distance between 3 and $4 \AA$ is required to form an excimer. The intramolecular energy transfer occurs over distances which are often an order of magnitude larger than those probed by the excimer. This distance, represented by the Förster radius, $R_{0}$, depends on the spectral properties of the Förster pair ${ }^{(5,6)}$ present in the polymer chain. The fluorescence quantum yield of $D(\phi)$ and the overlap integral $(J)$ of the absorption spectrum of $A$ and the emission spectrum of $D$ are mainly responsible for the value of $R_{\mathrm{o}}$. The efficiency of energy transfer has been used by the Lakowicz ${ }^{(7-11)}$ and Guillet ${ }^{(12-17)}$ groups to determine the distribution of distances between D-A pairs.

Many polyesters have repeating sequences of the form $\mathrm{Ar}-\mathrm{S}_{n}$, where $\mathrm{Ar}$ contains a rigid aromatic unit, $\mathrm{S}$ 


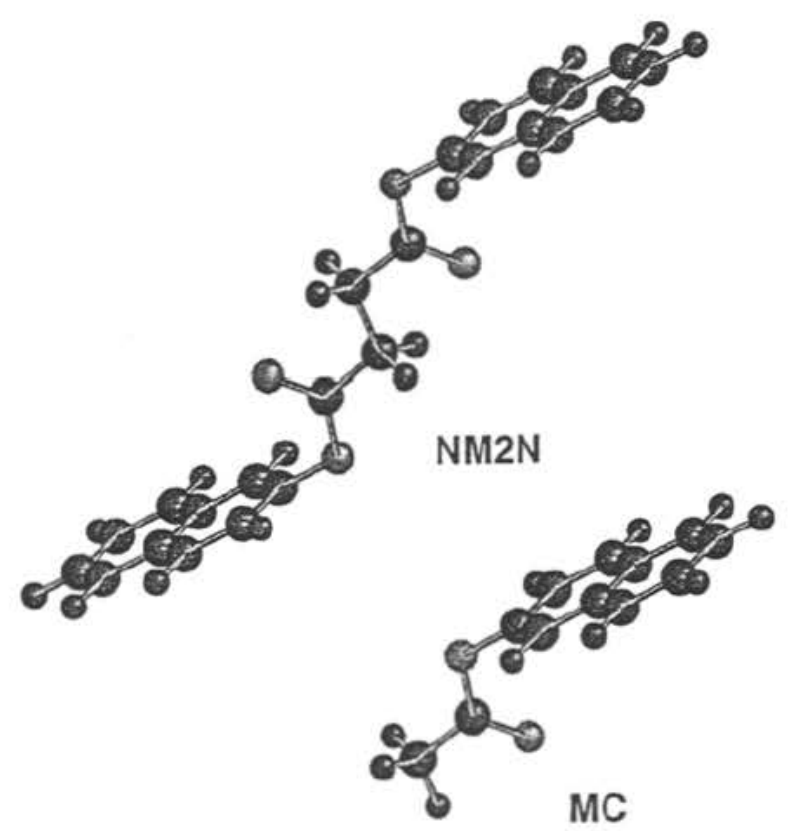

Fig. 1. Structures of NM2N and 2-naphthyl acetate (MC), with the former depicted in the starting conformation for the MD trajectory.

is a flexible spacer, and $n$ is a small positive integer. They exhibit a variety of properties depending on the manner in which Ar and S are chosen. A growing and interesting family of polyesters that have Ar units containing naphthalene ${ }^{(18-20)}$ is derived either from a naphthalene dicarboxylic acid and an aliphatic glycol ( $\mathrm{Ar}=\mathrm{Naph}-\mathrm{COO}$-) or from a dihydroxynaphthalene and an aliphatic dicarboxylic acid ( $\mathrm{Ar}=\mathrm{Naph}-\mathrm{OOC}$-). Conformation-dependent physical properties of polyesters with naphthalene units separated by flexible spacers depend on the directionality of the ester group, i.e., $\mathrm{N}$ $\mathrm{COO}-\mathrm{S}_{n}$ or $\mathrm{N}-\mathrm{OOC}-\mathrm{S}_{n} \cdot{ }^{(21-24)}$ Intramolecular excimer formation in bichromophoric model compounds, $\mathrm{Ar}-\mathrm{S}_{n}$ $\mathrm{Ar}$, gives information on how the probability for a sharp reversal (hairpin)(25) in the direction of the chain is influenced by the selection of this directionality of the ester group, the type of spacer, and $n$ (24) $^{(24)}$ efficiency of nonradiative singlet energy transfer (Förster transfer) also seems to be influenced.

The spectral properties that determine the efficiency of nonradiative singlet energy transfer in both series of naphthalene-containing polyesters have been described earlier. ${ }^{(21,22)}$ Nonradiative intramolecular energy transfer of polyesters (and their model compounds) derived from a naphthalene dicarboxylic acid and aliphatic glycols $^{(26-28)}$ (type Naph-COO-S - ), as well as model compounds where one naphthalene is replaced by an anthracene group, ${ }^{(28-30)}$ or two by pyrene groups, ${ }^{(31)}$ have been reported. The anisotropy of the fluorescence in a rigid medium, ${ }^{(26,27)}$ or extrapolated to a medium of very high viscosity, ${ }^{(31)}$ or simply the emission spectra at different viscosities ${ }^{(28-30)}$ provide information about the dependence of the energy transfer on $n$ for different D-A pairs. A theoretical treatment of the conformation of the flexible spacer, using the equilibrium RIS model, ${ }^{(32,33)}$ permits us to explain this dependence on the basis of the estimated values of $R_{0}$.

Here we study model compounds for the naphthalene-containing polyesters of the second series. The anisotropy was measured in a rigid medium for five bichromophoric compounds, obtained as condensation products of 2-naphthol and succinic, glutaric, adipic, pimelic, and suberic acids. Theoretical interpretation was carried out using molecular dynamics (MD) simulations. The value of $R_{0}$, which seems to be sensitive to the directionality of the ester group, is found to be in the range 9-10 $\dot{A}$. This value is smaller than the $R_{0}$ determined earlier for the nonradiative singlet energy transfers naphthoate $\rightarrow$ naphthoate, ${ }^{(26-28)}$ naphthoate $\rightarrow$ anthracenoate, ${ }^{(28-30)}$ and pyrenoate $\rightarrow$ pyrenoate, ${ }^{(31)}$ which are approximately 12,16 , and $21 \AA$, respectively. $\mathrm{MD}$ simulations support the experimental assignment of $R_{0}$ and rationalize the trend of the efficiency of the energy transfer with $n$.

\section{EXPERIMENT}

The bichromophoric compounds as a series are abbreviated $\mathrm{NM} n \mathrm{~N}$, and a specific member of the series is denoted by substitution of the number of methylene groups in the spacer for $n$. Synthesis, characterization, and purification of the $\mathrm{NMnN}$, where $n=2-6$, and the model compound 2-naphthyl acetate (MC) were described elsewhere. ${ }^{(22)}$ In brief, the diesters depicted in Fig. 1 were synthesized from 2-naphthol and the acid chlorides of succinic, glutaric, adipic, pimelic, and suberic acids, in the presence of triethylamine in chloroform at ambient temperature. MC was synthesized in the same way from 2-naphthol and acetyl chloride. After recrystallization several times from chloroform/methanol, the compounds were characterized by TLC, NMR, and ther$\mathrm{mal}$ analysis.

Dilute samples of the naphthalene containing molecules in glassy poly(methyl methacrylate) (PMMA) were prepared, as described previously, ${ }^{(26)}$ by the thermal polymerization of a very dilute solution (with an optical density in the range $0.3-0.5$ at $286 \mathrm{~nm}$ ) of the compounds in distilled methyl methacrylate, in the absence of an initiator. In brief, solutions of diesters and the 


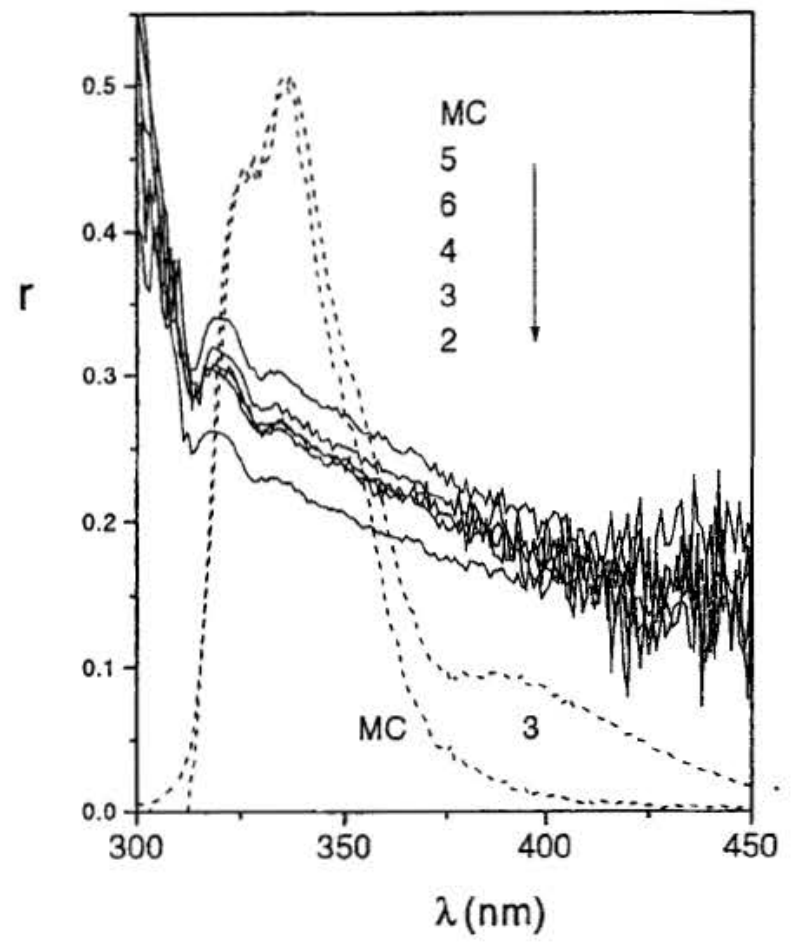

Fig. 2. Fluorescence anisotropy $(-)$ in a solid PMMA matrix at room temperature for $\mathrm{MC}$ and $\mathrm{NMnN}$, and normalized (at $335 \mathrm{~nm}$ ) emission spectra (---) for MC and NM3N in 1-propanol at $25^{\circ} \mathrm{C}$. Spectra for the $\mathrm{NMnN}$ are denoted by the value of $n$.

model compound were prepared in freshly distilled methyl methacrylate at the indicated concentration. Approximately $3 \mathrm{ml}$ of each solution was introduced into a 3-cm test tube, which was used as a mold. To remove the dissolved $\mathrm{O}_{2}$, the sample was placed in an ultrasonic bath for about $45 \mathrm{~min}$ and dry $\mathrm{N}_{2}$ was bubbled through the solution for about $10 \mathrm{~min}$. The polymerization was carried out in two steps. First, the temperature was increased slowly up to $70^{\circ} \mathrm{C}$ over approximately $4 \mathrm{~h}$. In the second step the temperature was increased to $90^{\circ} \mathrm{C}$, and this temperature was maintained for approximately $12 \mathrm{~h}$. The vitrified transparent samples were retrieved by breaking the test tube. All samples were prepared at the same time with the same experimental conditions.

Fluorescence depolarization measurements were performed at ambient temperature using an SLM 8100 AMINCO fluorometer, equipped with a cooled photomultiplier and a double monochromator in the excitation path. Slit widths were $4 \mathrm{~nm}$ for excitation and emission, and excitation was at $286 \mathrm{~nm}$. Front-face illumination, with the incident beam forming a $60^{\circ}$ angle with the surface of the solid sample to minimize the amount of light that reflects directly into the emission channel, was used for the fluorescence measurements.

\section{Anisotropy of the Fluorescence}

The anisotropy of the fluorescence, denoted $r$, was measured by the "L" method, as described by Lakowicz, ${ }^{(1)}$

$$
r=\left(I_{\mathrm{VV}}-G I_{\mathrm{VH}}\right) /\left(I_{\mathrm{VV}}+2 G I_{\mathrm{VH}}\right)
$$

where $I_{x y}$ is the intensity of the emission that is measured when the excitation polarizer is in position $x$ ( $V$ for vertical, $\mathrm{H}$ for horizontal), the emission polarizer is in position $y$, and $G=I_{\mathrm{HV}} / I_{\mathrm{HH}}$ corrects for depolarization produced by the optical system.

Figure 2 depicts the emission spectra for $\mathrm{MC}$ and $\mathrm{NM} 3 \mathrm{~N}$ and emission anisotropy spectra for $\mathrm{NMnN}$ and MC. Emission spectra are dominated by a band which occurs at $335 \mathrm{~nm}$ in PMMA at room temperature, as shown in Fig. 2. The emission spectra for $\mathrm{NM} n \mathrm{~N}$ are slightly broader to the red than the emission spectra for MC. This broadening is attributed to a weak signal from intramolecular excimers, for which the band is centered around $400 \mathrm{~nm}$. The intensity of this band depends on $n$, with the maximum value at $n=3^{(22,24)}$

There is a spectral region at approximately 315$375 \mathrm{~nm}$ where the emission has a substantial intensity (the monomer band) and the results for $r$ are more reliable. Outside of this range the anisotropy of the signals has a high noise and some of the values of $r$ are unreliable. These values are even larger than 0.4 at the low-wavelength region of the spectrum, where the emission is extremely weak. The decrease in $r$ as $\lambda$ increases, which takes place in all compounds in the same way, must be due to intrinsic causes of depolarization dependent on the optical conditions of the experiment, such as stray light or scattering by the PMMA matrix. The scattering is more effective at shorter wavelengths. If some of the scattered light is measured as fluorescence, the apparent anisotropy would increase. ${ }^{(35)} \mathrm{We}$ focus our interest on the relative sizes of $r$ for MC and the $\mathrm{NMnN}$. As expected, the largest value of $r$ is obtained with $\mathrm{MC}$, which contains a single chromophore. In the absence of any depolarization processes during the fluorescence lifetime, the value should be $2 / 5$ for a random orientation of independent chromophores. Smaller values are obtained for the bichromophoric compounds than for MC. In the whole range of measurement indicated, the values of $r$ follows a similar trend. As $n$ increases from 2 to $6, r$ varies as $2<3<4<6<5$.

Assuming that the depolarization observed for MC is due to intrinsic reasons, the experimental efficiency of energy transfer $\Phi_{\text {exp }}$ could be estimated as

$$
\Phi_{\text {exp }}=\left(r_{\mathrm{MC}}-r_{\mathrm{NMnN}}\right) / r_{\mathrm{MC}}
$$




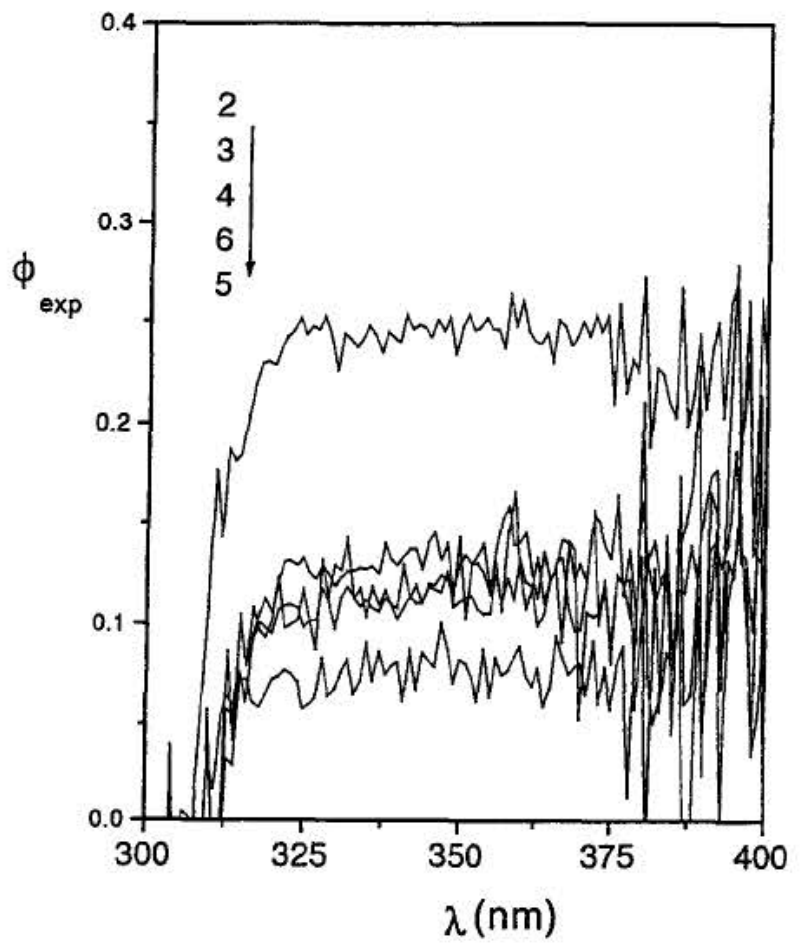

Fig. 3. Experimental efficiency of energy transfer for the NMnN, with each curve denoted by the value of $n$.

where $r_{\mathrm{MC}}$ and $r_{\mathrm{NMnN}}$ are the anisotropies observed at each wavelength for $\mathrm{MC}$ and $\mathrm{NM} n \mathrm{~N}$, respectively. Figure 3 depicts experimental efficiencies obtained using Eq. (2). The approximately constant values, in the range 315-375 nm, for each compound suggest an intrinsic depolarization as the reason for the low values observed for MC and the decrease in $r$ as $\lambda$ increases. This intrinsic depolarization will take place for all $\mathrm{NM} n \mathrm{M}$ in the same way. The results depicted in Fig. 3 show that the efficiency of energy transfer varies with $n$ as $2>3$ $>4>6>5$. Table I summarizes these results. The absolute values of $\Phi_{\exp }$ are irrelevant for our purpose, and we focus our interest here on the relative values of $\Phi_{\exp }$ for the NMnN.

\section{Estimation of $\boldsymbol{R}_{\mathbf{0}}$}

The Förster radius for a D-A pair is defined as ${ }^{(1-6)}$

$$
R_{0}{ }^{6}=9000 \ln 10 \kappa^{2} \phi_{\mathrm{D}} J / 125 \pi^{5} n_{0}{ }^{4} L
$$

where $n_{\mathrm{O}}$ is the refractive index of the medium at the wavelength of excitation, $\kappa^{2}$ is the orientation factor for the dipole-dipole interaction, $L$ is Avogadro's number, and $J$ is the overlap integral between the normalized fluorescence intensity by the donor, $I(\lambda)$, and the extinction
Table I. Average Values of $\phi_{\text {exp }}$ Obtained via Eq. (2) from $r$ Measured in the Range $315-375 \mathrm{~nm}$, Where the Emission Has a Substantial Intensity

\begin{tabular}{cc}
\hline$n$ & $\phi_{\text {exp }}$ \\
\hline 2 & $0.25 \pm 0.05$ \\
3 & $0.14 \pm 0.05$ \\
4 & $0.12 \pm 0.05$ \\
5 & $0.08 \pm 0.05$ \\
6 & $0.12 \pm 0.05$ \\
\hline
\end{tabular}

Table II. Values of Quantum Yields Overlap Integrals $J$ (in $M^{-1}$ $\mathrm{cm}^{3}$ ) for Naphthalene and 2-Naphthyl Acetate (MC) Measured in pDioxane, Methanol, and 1-Propanol at $25^{\circ} \mathrm{C}$

\begin{tabular}{lcccc}
\hline Solvent & $\phi_{\text {napheneiene }}$ & $\phi_{\text {MC }}$ & $10^{17} \times J_{\text {napthalene }}$ & $10^{17} \times J_{\text {MC }}$ \\
\hline -Dioxane & 0.10 & 0.14 & 3.41 & 13.9 \\
Methanol & 0.17 & 0.31 & 4.17 & 14.0 \\
1-Propanol & 0.07 & 0.10 & 2.87 & 12.6 \\
\hline
\end{tabular}

coefficient for the acceptor, $\varepsilon(\lambda) . J$ is defined as

$$
J=\int \lambda^{4} I(\lambda) \varepsilon(\lambda) d \lambda
$$

where, by definition,

$$
\int I(\lambda) d \lambda=1
$$

The ratio of the Förster radii for self-transfer by two molecules, $A^{*} \rightarrow A$ and $B^{*} \rightarrow B$, in the same environment can be written from Eq. (3) as

$$
\left(R_{0 \mathrm{~A}} / R_{0 \mathrm{~B}}\right)^{6}=\mathrm{\kappa}_{\mathrm{A}}^{2} \phi_{\mathrm{A}} J_{\mathrm{A}} / \mathrm{\kappa}_{\mathrm{B}}^{2} \phi_{\mathrm{B}} J_{\mathrm{B}}
$$

The quantum yields for fluorescence of donors were obtained by the procedure described previously, ${ }^{(34)}$ and $J$ was obtained by graphic integration of Eq. (4). A random distribution of isolated chromophores has $\kappa_{A}^{2} / \kappa_{B}^{2}=$ 1.

Measurements of $\phi$ and $J$ for naphthalene and MC in dilute solution, for which the values are collected in Table II, show that the ratios of quantum yields for fluorescence, $\phi_{\mathrm{MC}} d \phi_{\text {naphthalene, }}$ are $1.42,1.89$, and 1.42 in $p$ dioxane, methanol, and 1-propanol, respectively, at $25^{\circ} \mathrm{C}$. The ratio of the overlap integrals, $J_{\mathrm{MC}} J_{\text {naphthalene, }}$ are $4.08,3.35$, and 4.39 , respectively. Combining these effects, the ratios $R_{0}(\mathrm{MC}) / R_{0}$ (naphthalene) are 1.34 , 1.36 , and 1.36 in p-dioxane, methanol, and 1-propanol at $25^{\circ} \mathrm{C}$, respectively. Assuming the two tabulated values by Berlman of $R_{\mathrm{O}}$ of $7.35 \AA^{(36)}$ or $6.69 \AA_{,},{ }^{(3)}$ for the transfer from naphthalene to naphthalene, the estimated values of $R_{\mathrm{O}}$ for self-transfer by $\mathrm{MC}$ are in the range 9-10 $\AA$. We take this value to be the Förster radius for 


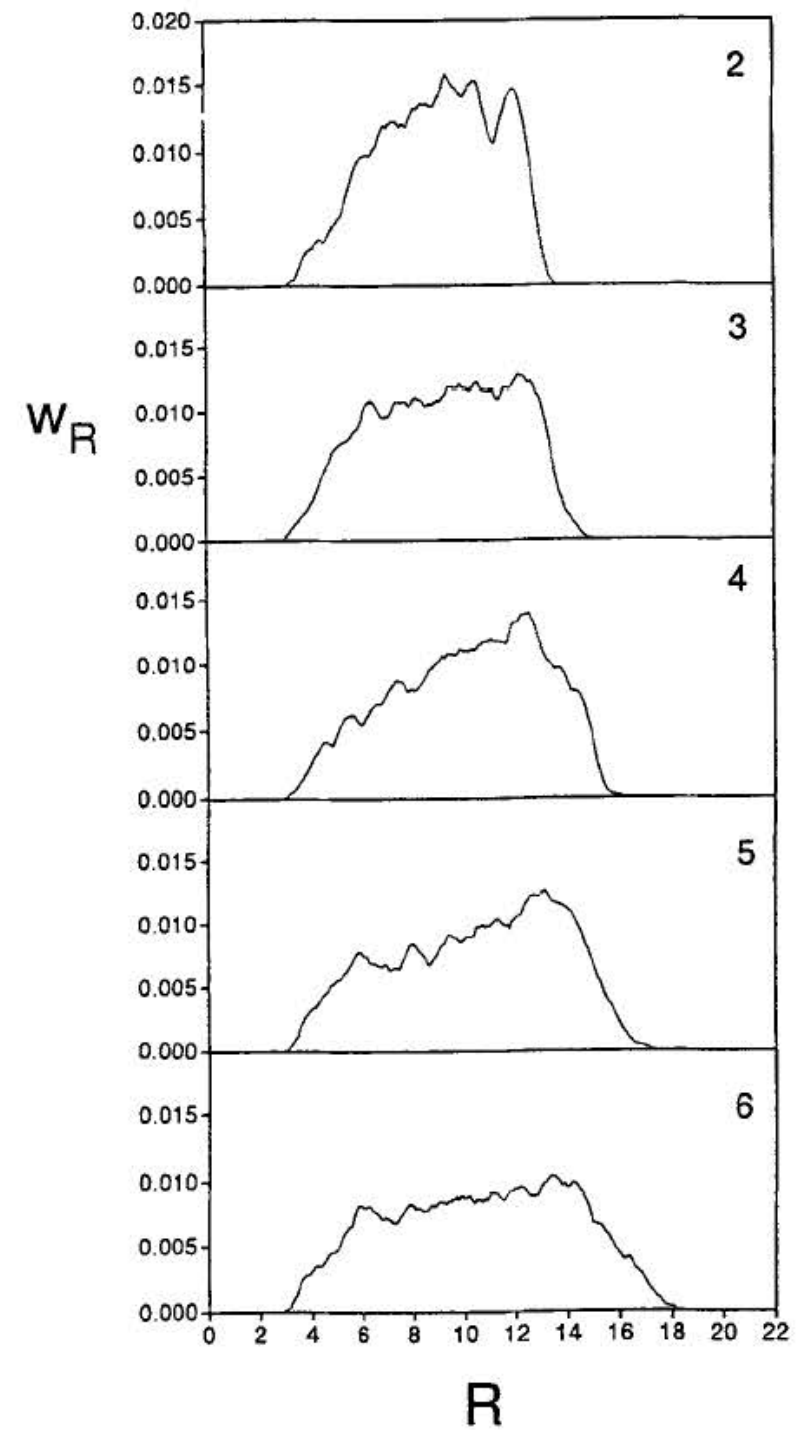

Fig. 4. Distribution of the distance between the centers of naphthalene groups for $\mathrm{NM} n \mathrm{~N}$. The value of $n$ is denoted.

the intramolecular energy transfer between naphthoxy groups in $\mathrm{NMnN}$.

\section{THEORETICAL}

\section{Methodology}

The MD trajectories of isolated molecules of $\mathrm{NM} n \mathrm{~N}$ were computed using Sybyl 6.0.(38) All bond lengths, bond angles, and torsion angles were variable in the simulations. The default Tripos force-field ${ }^{(39)}$ was employed and the electrostatic interactions were excluded from all simulations. The duration of each tra- jectory was $1 \mathrm{~ns}$, computed with a time step of $1 \mathrm{fs}$. Conformations were saved at intervals of $200 \mathrm{fs}$ for subsequent analysis, yielding 5000 conformations from each trajectory. The simulations were started on initial conformations where the flexible spacers were constructed with trans states at all internal rotatable bonds of the flexible spacer. The rigid naphthalene units were set to be perpendicular to the all trans skeleton of the spacer, which corresponds to a conformational minimum. The starting conformation for NM2N is depicted in Fig. 1. Each simulation began at $\mathrm{OK}$, and the temperature was increased by $10^{\circ}$ at intervals of $400 \mathrm{fs}$. Then the molecule was allowed a relaxation period at the temperature of interest for up to $100 \mathrm{ps}$ before the collection of data was initiated. Velocities were rescaled at intervals of 10 fs. A final temperature of $600 \mathrm{~K}$, which is high enough to sample a large region in conformational space and avoid entrapment in deep conformational energy wells, was used in all simulations.

\section{Parameters Related to the Energy Transfer Process}

Three parameters, denoted $p_{\mathrm{R}}, p_{\mathrm{R}} \mathrm{K}^{2}$, and $\Phi_{\text {teo, }}$, related to the amount of the energy transfer were obtained. ${ }^{(1-5)}$ Here $p_{\mathrm{R}}$ is the fraction of the time in the trajectory (or probability) for finding conformations where the centers of mass of the two chromophores are separated by a distance not larger than $R$,

$$
p_{\mathrm{R}}=\int w_{\mathrm{R}} d R
$$

where the integral extends from $\mathrm{O}$ to $R$, and $w_{\mathrm{R}}$ is the distribution function for the distance between the centers of the rings depicted in Fig. 4 for all five $\mathrm{NMnN}$. This distribution was obtained by equally weighting all conformations. As expected, the maximum of the distribution is shifted to higher distances and the average of the distances $\langle R\rangle$ between the centers of the naphthalene rings increases as $n$ increases. These average values are $9.06,9.23,10.14,10.39$, and $10.54 \AA$ as $n$ goes from 2 to 6 . Figure 5 depicts the history of the distance between centers of naphthalene rings during the 1-ns trajectory for NM2N and NM5N.

Figure 6 depicts the dependence of $p_{\mathrm{R}}$ on $n$ for different values of $R$. The value of $p_{\mathrm{R}}$ must equal 1 when $R$ exceeds the distance between the centers of the naphthalene rings in the fully extended conformation. Values of approximately 14 and $18 \AA$ are required for the compounds with $n=2$ and 6 , respectively, to reach $p_{\mathrm{R}}=$ 1. The values of $p_{\mathrm{R}}$ exhibit a weak odd-even effect at $R$ $<7 \AA$. In the range of $9 \AA \leq R \leq 11 \AA$ there are a decrease in $p_{\mathrm{R}}$ as $n$ increases from $n=2$ to 5 and a slight increase in the values of $p_{\mathrm{R}}$ for NM6N with respect 

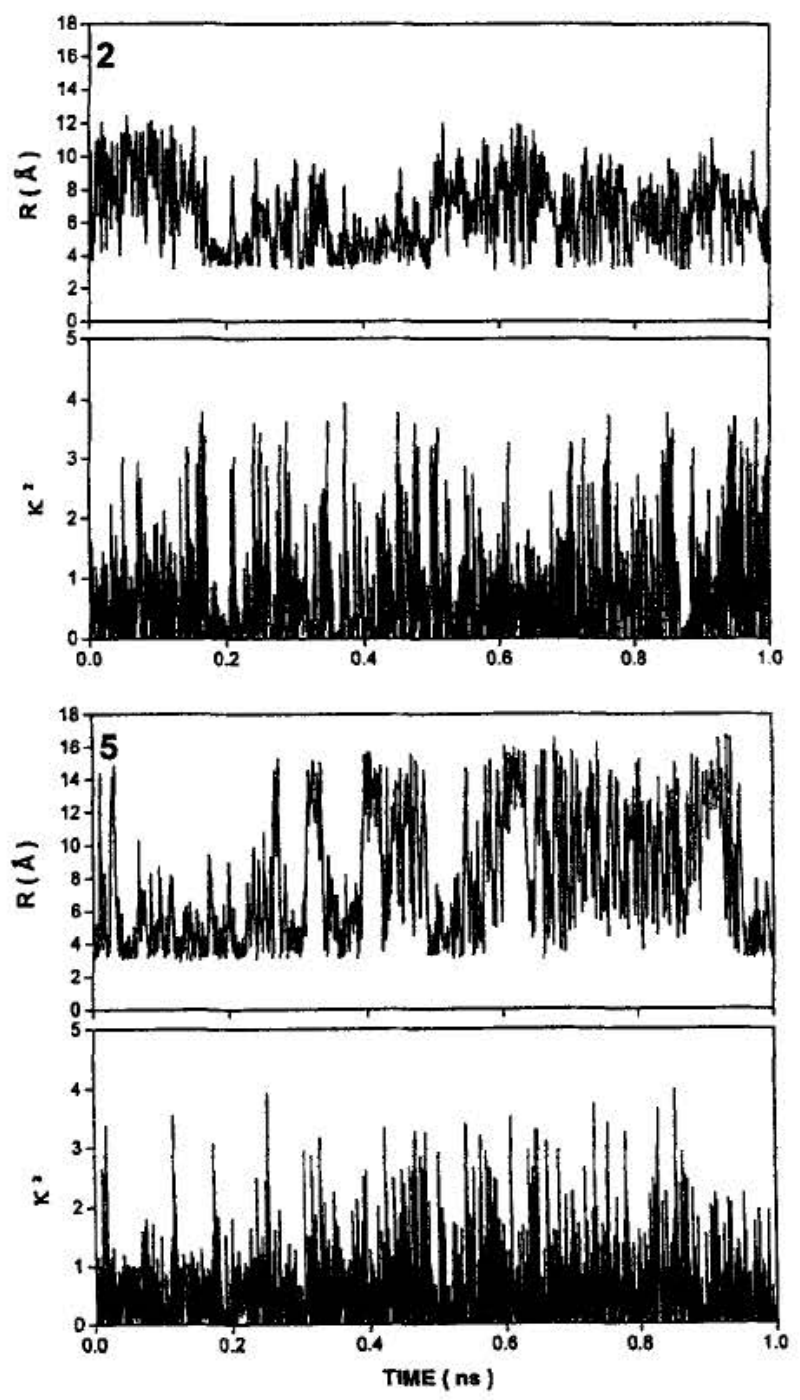

Fig. 5. History for the distance between centers of naphthalene rings and $\kappa^{2}$ for NM2N and NM5N during the 1-ns trajectory. The value of $n$ is denoted in each panel.

to NM5M. As $R$ reaches higher values, $12 \AA \leq R$, there is a continuous decrease in $p_{\mathrm{R}}$ as $n$ increases.

The product $p_{\mathrm{R}} \mathrm{K}^{2}$ as a function of $n$ was also calculated in a similar manner. The value of this product was then normalized for each compound. As with $p_{R}$, $p_{\mathrm{R}} \kappa^{2}$ must approach 1 as $R$ becomes higher than the maximum distance between centers of the naphthalene rings for each compound. The trends of $p_{\mathrm{R}} \mathrm{K}^{2}$ (not shown) and $p_{\mathrm{R}}$ (Fig. 6 ) with $n$ for different $R$ are very similar. The values of $p_{\mathrm{R}} \kappa^{2}$ exhibit the odd-even effect for $R<7 \AA$, whereas the continuous decrease with $n$ is reached for $R$ $>12 \AA$. A continuous decrease as $n$ increases form $n$ $=2$ to 5 and a slight increase in the values of $p_{\mathrm{R}} \mathrm{K}^{2}$ for NM@N with respect to NM5N are obtained for $9 \AA<$

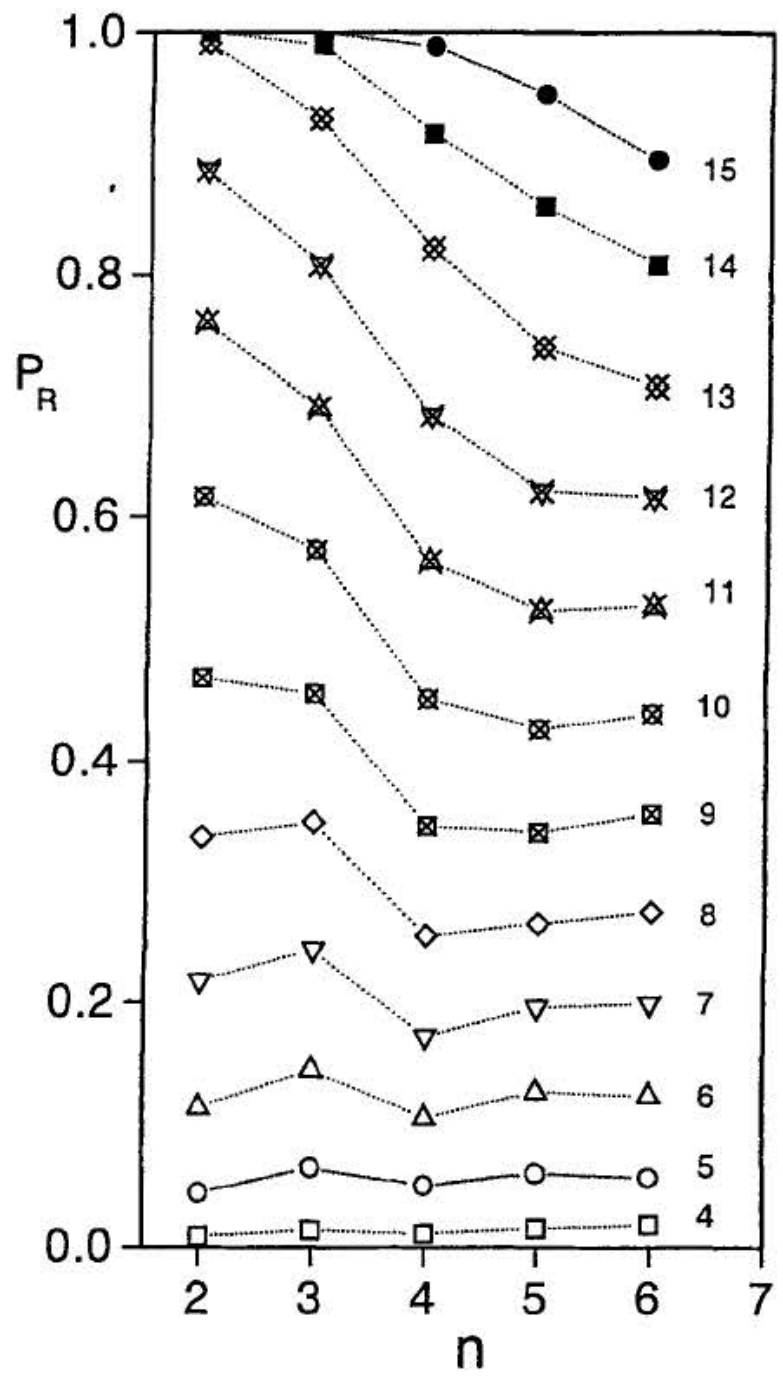

Fig. 6. $p_{\mathrm{R}}$ for $\mathrm{NMnM}$ for $R$ ranging from 4 to $15 \AA$, at intervals of 1 $\AA$.

$R<11 \AA$. The averages of $\kappa^{2}$, denoted $\left\langle\kappa^{2}\right\rangle$, are 0.61 , $0.59,0.63,0.62$, and 0.60 for the $\mathrm{NM} n \mathrm{~N}$ with $n=2,3$, 4,5 , and 6 , respectively. These values are slightly smaller than the expectation (2/3) for a random distribution of chromophores and were evaluated as

$$
\left\langle\kappa^{2}\right\rangle=(1 / N) \Sigma \kappa_{i}^{2}
$$

where the sum is extended to all recorded conformations $N\left(=5000\right.$ conformations) and $\kappa_{i}^{2}$ is the orientational factor for conformation $i$. Figure 5 depicts instantaneous values for $k^{2}$ obtained for NM2N and NM5N.

A third parameter is $\Phi_{100}$, the efficiency of Förster transfer energy for several assumptions about the value of $R_{0}$, obtained according to

$$
\Phi_{\text {too }}=(1 / N) \Sigma\left[1+\mathrm{\kappa}^{2} R^{6} /(2 / 3) R_{0}^{6}\right]^{-1}
$$




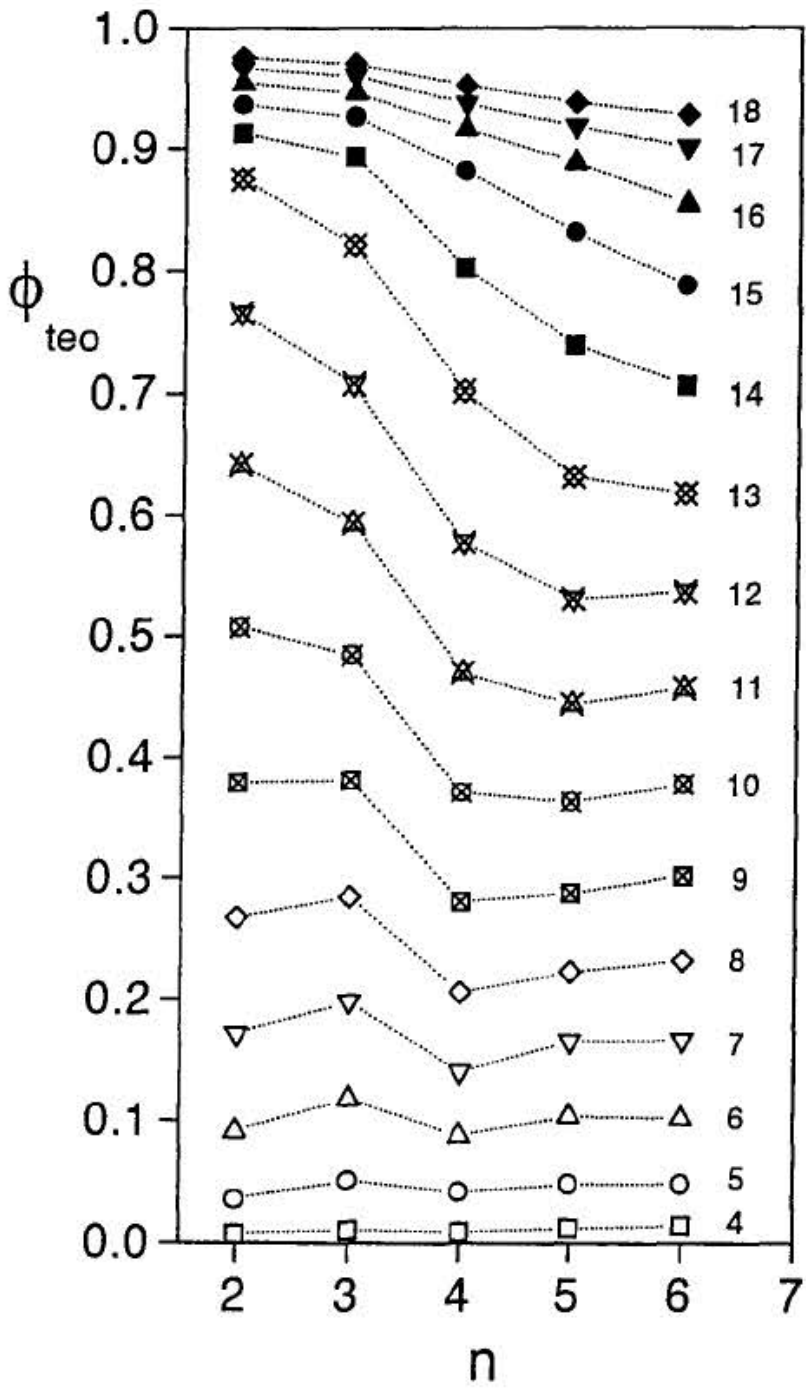

Fig. 7. Efficiency of Förster transfer for several assumptions about the value of $R_{0}(4-18 \AA$, at intervals of $1 \AA)$ for $\mathrm{NMnN}$.

where the sum extends over all conformations, $R$ is the known distance between the centers of the naphthalene rings, and $R_{\mathrm{O}}$ is the assumed value for the Förster radius. Figure 7 depicts the $\Phi_{\text {teo }}$ obtained by Eq. (9) as a function of $n$ and $R_{0}$ for the $\mathrm{NMnN}$. The continuous decrease in the $\Phi_{\text {teo }}$ for $n=2-5$ and the slight increase in $\Phi_{\text {teo }}$ for NM6N occurs in the range $10 \AA \leq R \leq 12 \AA$. An odd-even trend and a continuous decrease for $n=2-6$ are obtained for $R<7 \AA$ and $R>13 \AA$, respectively.

\section{DISCUSSION}

Our experimental estimate of $R_{\mathrm{o}}$ for the intramolecular energy transfer between naphthoxy groups in
$\mathrm{NM} n \mathrm{~N}$ was around $10 \AA$. This value is about $2 \AA$ smaller than the value obtained for the transfer from naphthoate to naphthoate, ${ }^{(26.27)}$ where the ester group is reversed, and larger than the values tabulated by Berlman of $7.35 \AA^{(35)}$ and $6.69 \AA^{(36)}$ for the transfer from naphthalene to naphthalene. This value of $R_{0}$, in the range 9-10 $\AA$, is consistent with the trend of the experimental data for the efficiency of the energy transfer process $\Phi_{\text {exp }}$, collected in Table I, and the theoretically calculated parameters $p_{\mathrm{R}}$ and $\Phi_{\text {exp }}$ with $n$ depicted in Figs. 6 and 7. Experimental anisotropy results show that $\Phi_{\exp }$ varies with $n$ as $2>$ $3>4>6>5$. The estimated value of $R_{\mathrm{O}}$ in the range 9-10 $\AA$ in either of Fig. 6 or Fig. 7 reproduces satisfactorily the experimental trend of the efficiency of energy transfer with $n$ and demonstrates the expected conformational dependence of the intramolecular energy transfer, which requires adequate distance and orientation of a donor-acceptor pair linked with short flexible chains.

\section{ACKNOWLEDGMENTS}

This research was supported by Grants DGICYT PB94-0364 and UAH 017/95 (A.S.-C., J.P., and F.M.) and by NSF Grant DMR 9523278 (W.L.M.)

\section{REFERENCES}

1. J. R. Lakowicz (1983) Principles of Fluorescence Spectroscopy, Plenum Press, New York.

2. J. Guillet (1985) Polymer Photochemistry and Photophysics: An Introduction to the Study of Photoprocesses in Macromolecules, Cambridge University Press, Cambridge.

3. D. Phillips (Ed.) (1985) Polymer Photophysics: Luminescence, Energy Migration and Molecular Motion in Synthetic Polymer, Chapman and Hall, London.

4. J. F. Rabeck (1987) Mechanisms of Photophysical Processes and Photochemical Reactions in Polymers: Theory and Applications, John Wiley and Sons, Chichester.

5. Th. Förster (1948) Ann. Phys. 2, 55.

6. I. B. Berlman (1973) Energy Transfer Parameters of Organic Compounds, Academic Press, New York.

7. A. S. Jones, T. J. Dickson, B. E. Wilson, J. Duhamel, and M. A. Winnik (1996) Polym. Prepr. (Am. Chem. Soc. Div. Polym. Chem.) 37(1), 229.

8. J. R. Lakowicz, I. Gryczynski, H. C. Cheung, C.-K. Wang, and M. L. Jhonson (1988) Biopolymers 27, 821.

9. I. Gryczynski, W. Wiczk, M. L. Jhonson, H. C. Cheung, C.-K. Wang, and J. R. Lakowicz (1988) Biophys. J. 54, 577.

10. I. Gryczynski, W. Wiczk, M. L. Jhonson, and J. R. Lakowicz (1988) Chem. Phys. Lett. 145, 439.

11. J. R. Lakowicz, J. Kusba, H. Szmacinski, I. Gryczynski, P. S. Eis, W. Wiczk, and M. L. Jhonson (1991) Biopolymers 31, 1363.

12. J. R. Lakowicz, W. Wizck, I. Gryczynski, M. Fishman, and M. L. Jhonson (1993) Macromolecules 26, 349.

13. G. Liu and J. E. Guillet (1990) Macromolecules 23, 1388.

14. G. Liu, J. E. Guillet, E. T. B. Al-Takrity, A. D. Jenkins, and D. R. M. Walton (1990) Macromolecules 23, 1393.

15. G. Liu and J. E. Guillet (1990) Macromolecules 23, 2969. 
16. G. Liu, J. E. Guillet, E. T. B. Al-Takrity, A. D. Jenkins, and D. R. M. Walton (1990) Macromolecules 23, 4165.

17. G. Liu, J. E. Guillet, E. T. B. Al-Takrity, A. D. Jenkins, and D. R. M. Walton (1991) Macromolecules 24, 68.

18. G. Liu, J. E. Guillet, M. Vlegels, and E. J. Goethals (1991) Macromolecules 24, 4094.

19. M. Guo (1996) Polym. Prepr. (Am. Chem. Soc. Div. Polym. Chem.) 37(1), 227, 827.

20. M. Guo and H. G. Zachmann (1996) Polym. Prepr. (Am. Chem. Soc. Div. Polym. Chem.) 37(1), 825, 829, 831.

21. F. Mendicuti, B. Patel, and W. L. Mattice (1990) Polymer 31, 453.

22. F. Mendicuti, E. Saiz, I. Zúñiga, B. Patel, and W. L. Mattice (1992) Polymer 33, 2031.

23. O. Martin, F. Mendicuti, E. Saiz, and W. L. Mattice (1996) J. Polym. Sci. Part B Polym. Phys. 34, 2623.

24. O. Martin, A. Sanchez-Camacho, F. Mendicuti, and W. L. Mattice (1997) J. Polym. Sci. Part B Polym. Phys. 35, 1127.

25. P. G. de Gennes (1982) in A. Ciferri, W. R. Krigbaum, and R. B. Meyer (Eds.) Polymer Liquid Crystals; Academic Press, New York, Chap. 8.

26. F. Mendicuti, E. Saiz, and W. L. Mattice (1992) Polymer 33, 4908.
27. J. Gallego, F. Mendicuti, E. Saiz, and W. L. Mattice (1993) Polymer 34, 2475.

28. F. Mendicuti, E. Saiz, J. Bravo, and W. L. Mattice (1995) Polym. Int. 36, 137.

29. J. Bravo, F. Mendicuti, E. Saiz, and W. L. Mattice (1994) Makromol. Chem. 195, 3411 .

30. J. Bravo, F. Mendicuti, E. Saiz, and W. L. Mattice (1996) Macromol. Chem. Phys. 197, 1349.

31. J. Bravo, F. Mendicuti, E. Saiz, and W. L. Mattice (1996) J. Fluoresc. 6,41 .

32. P. J. Flory (1974) Macromolecules 7, 381.

33. W. L. Mattice and U. W. Suter (1994) Conformational Theory of Large Molecules. The Rotational Isomeric State Model in Macromolecular Systems, Wiley, New York.

34. F. Mendicuti and W. L. Mattice (1989) Polym. Bull. 22, 557.

35. J. R. Lakowicz (1983) Principles of Fluorescence Spectroscopy, Plenum Press, New York, p 134.

36. I. B. Berlman (1973) Energy Transfer Parameters of Organic Compounds, Academic Press, New York, p 308.

37. I. B. Berlman (1973) Handbook of Fluorescence Spectra of Aromatic Molecules, Academic Press, New York, p. 421.

38. Sybyl 6.0, Tripos Associates, Inc., St. Louis, MO

39. M. Clark, R. D. Cramer, III, and N. Van Opdenbosch (1989) Comput. Chem. 10, 982. 